\title{
Parts of the Whole: An Algebra Lesson
}

\section{Dorothy Wallace}

Dartmouth College, dorothy.wallace@dartmouth.edu

Follow this and additional works at: https://digitalcommons.usf.edu/numeracy

Part of the Mathematics Commons, and the Science and Mathematics Education Commons

\section{Recommended Citation}

Wallace, Dorothy. "Parts of the Whole: An Algebra Lesson." Numeracy 4, Iss. 2 (2011): Article 10. DOI: http://dx.doi.org/10.5038/1936-4660.4.2.10 


\title{
Parts of the Whole: An Algebra Lesson
}

\begin{abstract}
This column draws on research of Eon Harper to demonstrate how an understanding of his proposed stages of algebra acquisition would inform a systemic overhaul of algebra education. Harper's stages also explain why students may pass a series of algebra courses yet still be unable to make sense of calculus, as well as offering insight on what aspects of algebra support quantitative literacy.
\end{abstract}

\section{Keywords}

algebra, cognitive stage, educational system

\section{Creative Commons License}

\section{(c) (i) (2)}

This work is licensed under a Creative Commons Attribution-Noncommercial 4.0 License

\section{Cover Page Footnote}

Dorothy Wallace is a professor of mathematics at Dartmouth. She was 2000 New Hampshire CASE Professor of the Year, and the lead PI of the seminal NSF project, Mathematics Across the Curriculum. She recently finished a text in mathematical biology for first-year students, "Situated Complexity." She was a charter board member of the National Numeracy Network and is now co-editor of this journal. 


\section{Parts of the Whole}

\section{A Column by D. Wallace}

The problem of how best to improve the numeracy of a society is a thorny one, embracing the learning process of a single student but rising in scale to include the management and alteration of an entire system of education. With the issue of quantitative literacy always in mind, this column considers various aspects of the systemic workings of education, the forces acting on classrooms, teachers and students, and mechanisms of both stasis and change.

\section{An Algebra Lesson}

This column attempts to synthesize the material we have explored so far by using it to understand a single example. We have chosen algebra because its cognitive stages are somewhat understood, because it is the subject of intense debate in education, and because data on student performance are available. The analysis here is intended as a model for how one might approach any subject currently taught at any level, although the questions surrounding algebra are interesting in their own right.

Algebra began the way many parts of mathematics began: as a problem set. Greek mathematicians solved easy algebra problems without the kind of formal notation that we now recognize as an essential aspect. The mathematicians of Islam made great advances in algebra, using it to solve tricky legal problems of inheritances. The taint of Islam kept algebra from getting its full due in the early Renaissance, but eventually algebra problems became the fodder of mathematical "jousts" between different courts. The notation began to be solidified into the $x$ 's and $y$ 's we use commonly today. The French lawyer Viète made a huge advance by introducing the idea of a parameter, denoting constants in equations by letters so that whole classes of them could be solved at once. To this, Descartes added the coordinate plane that we use for graphing. The algebra we expect students to learn before they get to college took millennia to develop.

Algebra began as a purely mathematical exercise, yet it became the language necessary for doing science of all sorts. Nearly every relationship of importance in science can be summarized in a mathematical expression requiring an understanding of algebra. All statements about rates of growth and change are part of calculus, which in turn rests upon a firm fluency with algebra. Because the economic health of the nation rests to a large extent on continuing scientific advances, growing a suitable number of scientists is an important goal of the 
educational system. Under pressure to produce scientists, colleges and universities have made mathematical achievement, and in particular algebra, an entrance requirement.

\section{Variation in algebra acquisition}

Precisely because of the wide variation in mastery among students taking algebra, the mathematics score on the SAT exam is given great weight in college admissions. This score distinguishes students quite effectively, turning the understanding of algebra into a social marker that the student carries for many years. It is no wonder that Bob Moses, who founded "The Algebra Project", treats the matter as a civil rights issue, explicitly stating the goal of "algebra for all."

The true variation in algebra acquisition goes far beyond what the SAT can measure, ranging from those who never take algebra or the SAT, all the way to those who have mastered far more than what the SAT tests. This enormous spread has led to many suggestions. Some suggest teaching simpler concepts as early as the fourth grade, using hands-on methods typical for that age level. Some favor concentrating on arithmetic until almost college age. Some criticize the very push to algebra and suggest instead a focus on "quantitative literacy" or "practical mathematics," a problematic alternative when algebra mastery is necessary for doing most, if not all, science. Algebra education is an example of a system with such wide variance in output that it is difficult to believe it is in statistical control at all.

Algebra is an excellent example of a first-priority subject, because it usually requires the presence of a teacher over an extended time period (usually multiple years) to learn. Because algebra acquisition is so dependent upon the educational system, it is fair to assume that the system itself is driving the variation skyward. To analyze a system we must break it into component parts and look at how each part works. The key to understanding the components of the algebra conundrum is to revisit the fact that algebra is difficult, and ask why this is so.

\section{You have a system for learning algebra}

Eon Harper's research ${ }^{2}$ shows that there are at least three distinct schemas for understanding algebra and these are sorted into a strict hierarchy of acquisition. In other words, it is absolutely necessary to have the simplest schema intact before acquiring the second one, and the second one must be intact before

\footnotetext{
${ }^{1}$ Moses, R. and Cobb, C. Radical Equations: Civil Rights from Mississippi to the Algebra Project (Beacon Press, 2001)

${ }^{2}$ Harper, E. "Ghosts of Diophantus", Educational Studies in Mathematics Volume 18, Number 1, 75-90, 1987, Reidel
} 
proceeding to the third. So, the process of teaching algebra has at least three subprocesses that must be studied separately. These form the vertical spine of the "cognitive pyramid" described in our last column, and each of these three schemas must be reinforced sideways by connections with other knowledge. Those connections include bridges to some applications that certainly fit the usual definitions of quantitative literacy.

A few implications of Harper's research are immediately obvious. Suppose a teacher is introducing material in an algebra class that requires the development of the third and highest schema to understand. Suppose further that some of the students in the class haven't yet gotten the middle schema intact. All their attempts to make sense out of the material will be doomed to failure. It is not the fault of the teacher or the student-it is purely the fault of the system that requires these students to address this material at this particular time.

Another implication of these stages can be framed in terms of language. Suppose the teacher has a group of students with the first cognitive stage intact and wishes to move them to the next stage. The teacher may offer appropriate problems and explanations, yet those explanations may be expressed in sentences that make sense only if the student has the third and most abstract stage of cognition intact. Textbooks in particular are guilty of this kind of communication. The result is that the instructor or text appears to be speaking nonsense. The student may even believe that the instructor is being intentionally confusing. The problem is not that the instructor knows too little-on the contrary, the teacher knows too much and is using it too early. Again, without a comprehensible, managed system for learning algebra, this can hardly be said to be the teacher's fault.

\section{Harper's stages of algebra cognition}

According to Harper, the first and simplest cognitive stage in algebra is one where simple equations in a single variable are to be solved for the value of the variable. Loosely speaking, the variable isn't really a variable: it is a number that is concealed by a mask. The issue is always to find this number; there is usually an algorithm to do so, and the equal sign is generally interpreted as an injunction to compute something.

The second stage, which Harper calls "Diophantine" after a class of problems studied by Diophantus, is characterized by algebraic equations that are not intended to be solved for a single number. Equations involving several variables fall into this category, as do problems where what one wishes to know is more complex than the mere value of a variable. To answer the question requires viewing the entire equation as a relationship that can be re-expressed in various ways using algebraic manipulations. The equal sign is no longer the injunction to compute. It now serves as a fulcrum on which both sides of the equation balance. 
The variable need not be a single number that we do not know. There is rarely a well-defined algorithm that works for most such problems.

The third stage is what Harper calls "Viètan," after the French mathematician and lawyer mentioned earlier. When Viète introduced alphabetic notation for the actual constants in an equation, he allowed a new thing to happen. Now, instead of looking at a single equation, we can study whole classes of them. The equation now becomes an object in its own right, and equations can be sorted and classified according to various properties. General formulas, like the "quadratic formula," can express answers for infinitely many problems in full generality at once.

The ability to look at a class of examples, extract a general property, name it, and use it, requires a conceptual advance. Harper's research on schoolboys in England showed that a minority of those graduating from secondary school had acquired this schema. It is a difficult schema, yet it is impossible to understand a calculus text, for example, without being able to think in these terms. Harper devised a diagnostic test that would allow him to see which schemas were intact in each individual. The fact that we make no use of such a test when attempting to teach algebra is an indication that, not only is our system of algebra education poorly managed, it is not managed at all. Most diagnostic tests, like the SAT, contain problems that can be worked in each of the three schemas. It is possible, therefore, to obtain passing scores on these kinds of tests year after year without ever developing the cognitive structure necessary to proceed to calculus. A student entering calculus with such an incomplete background will not be able to read the text with understanding, nor will any lecture be completely comprehensible. If enough of the calculus test problems are strictly algorithmic in nature, the student may still memorize enough to pass and continue to the next course, but this is a debatable victory at best.

We might ask what sorts of real-world tasks correspond to each of the three schemas. The simplest schema is really just generalized arithmetic. It allows us to handle a few more problems than before with a more elegant language. It allows us to program a graphing calculator and understand what that calculator is doing to produce its picture. It allows someone to make good use of an Excel spreadsheet. The second schema is richer. It allows one to write down more complicated relationships among quantities, such as those that occur in geometric contexts. It allows us to do simple science, working with equations that have more than one variable (like pressure, temperature and volume of a gas). The third schema is necessary for calculus and for any activity that will require serious mathematical analysis. This category includes all serious science as well as economics and certain types of social science.

\section{Schemas of the algebra teacher}

It is worth noting how people with all three schemas intact typically function. My description here is based on personal experiments in our department teaching 
seminar. Participants, who are all fairly advanced in mathematics, are asked to explain a simple algebraic expression as they would to a child in second grade, a somewhat more complex example to an older child, and finally a mathematical expression using parameters and quantifiers to a pre-calculus student. What people do in this case is very consistent. They use the simplest schema necessary to explain whatever they are doing at the moment. The result is usually a beautiful delineation among the three schema identified by Harper.

The calculus instructor, in particular, will have all of these schemas at his or her disposal, and will be inclined to use the simplest schema necessary to explain whatever topic is under discussion at the moment. Many problems in calculus are somewhat algorithmic in nature, so the first schema gets used fairly frequently. The general rules of calculus are really only comprehensible if the third schema is invoked, however. The middle schema gets used a fair amount also. What is the effect on the listener who only has the first two schemas intact? A certain proportion of the explanation given makes absolutely perfect sense. The rest of it is mere gibberish. There are two likely conclusions on the part of the student: "I am stupid" or "This instructor is awful," perhaps both.

\section{Some implications}

How, then, might we approach the algebra problem? To reduce variation among students and proceed in an orderly manner, it would make sense to have three separate algebra courses, each addressing one and only one of the cognitive levels. A student passing such a course should have the corresponding level intact. Other courses with algebra prerequisite would have to be scrutinized in order to find the minimal level of algebra necessary for succeeding in them. Courses that make use of algebra at a particular level should be introduced as early as possible, in order to extend the early schemas and strengthen them. The third course in the algebra sequence must be dedicated to forming that third, most difficult, schema. A diagnostic test at the beginning would indicate if the students were ready to tackle the material. The teacher would ruthlessly demand that every student function at that level for every single test problem. Perhaps an additional course could bolster previous schema through a variety of applications, so that the "cognitive pyramid" would grow wider as well as taller.

This series of suggestions is quite different from what is currently done. Every algebra course attempts to teach material from at least two if not all three cognitive levels, without regard to whether the students have mastered the previous one or not. Weak students take repeated versions of this course, succeeding and failing repeatedly at the same points. Eventually a student may pass the course, but in terms of cognition nobody will know what that means. By shooting the same gun at the same target over and over again we can never reduce variance, change the mean, or improve the system. If, on the other hand, we 
break the large problem of learning algebra into appropriate sub-processes, we can work on each of the smaller processes separately to improve it.

These processes also provide a clue to how we might answer the question of whether algebra is necessary. Would we like everyone in our society to be able to run an Excel spreadsheet? If so, then the first level of algebraic understanding is necessary to everyone. How many scientists do we need? At least twice that many students ought to be achieving the highest cognitive level in algebra. Do we want our students to remember the things they learn? Then we must find ways to widen the cognitive pyramid, because connected information is better retained and better utilized. Courses stressing quantitative literacy that use simple algebra effectively will help reach that goal.

The analysis of the algebra question has implications for teacher preparation as well. A teacher who does not understand the structure of cognitive stages in algebra would be unable to teach effectively the courses suggested in this column. A teacher whose Viètan schema was not yet complete would be unable to teach the third course at all. Treating education as a system implies that we would pay attention to the unique needs of each part of the process. Teaching algebra with regard to the issues discussed here requires a person with specialized knowledge and expertise. Having passed an algebra course will hardly suffice. The algebra problem is systemic and requires intervention in teacher preparation as well in order to succeed. Algebra is a prime example of how careful, informed analysis of the educational process has the potential to improve the system enormously. 